\title{
Many-Body Dynamics of Dipolar Molecules in an Optical Lattice
}

\section{Citation}

Hazzard, Kaden R. A., Bryce Gadway, Michael Foss-Feig, Bo Yan, Steven A. Moses, Jacob P. Covey, Norman Y. Yao, et al. 2014. "Many-Body Dynamics of Dipolar Molecules in an Optical Lattice." Physical Review Letters 113 (19) (November). doi:10.1103/physrevlett.113.195302.

\section{Published Version}

doi:10.1103/PhysRevLett.113.195302

\section{Permanent link}

http://nrs.harvard.edu/urn-3:HUL.InstRepos:16953021

\section{Terms of Use}

This article was downloaded from Harvard University's DASH repository, and is made available under the terms and conditions applicable to Other Posted Material, as set forth at http:// nrs.harvard.edu/urn-3:HUL.InstRepos:dash.current.terms-of-use\#LAA

\section{Share Your Story}

The Harvard community has made this article openly available.

Please share how this access benefits you. Submit a story.

Accessibility 


\title{
Many-Body Dynamics of Dipolar Molecules in an Optical Lattice
}

\author{
Kaden R. A. Hazzard, ${ }^{1, *}$ Bryce Gadway, ${ }^{1}$ Michael Foss-Feig, ${ }^{2}$ Bo Yan, ${ }^{1}$ Steven A. Moses, ${ }^{1}$ Jacob P. Covey, \\ Norman Y. Yao, ${ }^{3}$ Mikhail D. Lukin, ${ }^{3}$ Jun Ye, ${ }^{1}$ Deborah S. Jin, ${ }^{1}$ and Ana Maria Rey ${ }^{1}$ \\ ${ }^{1}$ JILA, National Institute of Standards and Technology and University of Colorado, Department of Physics, \\ University of Colorado, Boulder, Colorado 80309-0440, USA \\ ${ }^{2}$ Joint Quantum Institute, National Institute of Standards and Technology, and University of Maryland, \\ Gaithersburg, Maryland 20899, USA \\ ${ }^{3}$ Physics Department, Harvard University, Cambridge, Massuchusetts 02138, USA
}

(Received 3 June 2014; published 7 November 2014)

\begin{abstract}
We use Ramsey spectroscopy to experimentally probe the quantum dynamics of disordered dipolarinteracting ultracold molecules in a partially filled optical lattice, and we compare the results to theory. We report the capability to control the dipolar interaction strength. We find excellent agreement between our measurements of the spin dynamics and theoretical calculations with no fitting parameters, including the dynamics' dependence on molecule number and on the dipolar interaction strength. This agreement verifies the microscopic model expected to govern the dynamics of dipolar molecules, even in this strongly correlated beyond-mean-field regime, and represents the first step towards using this system to explore many-body dynamics in regimes that are inaccessible to current theoretical techniques.
\end{abstract}

DOI: 10.1103/PhysRevLett.113.195302

Advances in trapping and cooling ground-state polar molecules have produced ultracold, nearly degenerate gases [1-4], allowed complete control of their hyperfine, rotational, vibrational, and electronic degrees of freedom $[5,6]$, and enabled their preparation in optical lattices [7]. Long-range dipolar interactions among molecules facilitate the exploration of fascinating many-body phenomena [8-13] and are useful for quantum information processing [14]. By encoding a spin- $1 / 2$ degree of freedom in two rotational states, Ref. [15] reported the first observation of dipolar-exchange interactions between molecules in a three-dimensional optical lattice, with signatures such as density dependence of the spin coherence dynamics as probed by Ramsey spectroscopy (Fig. 1). Although such dynamics is expected to be governed by a particular spin model [16,17], a quantitative demonstration that the experimental observations are consistent with this model was lacking. Such a demonstration requires comparison of the experimental measurements with theoretical calculations of this three-dimensional correlated system during probing times long enough to resolve the long-ranged anisotropic dipolar interactions.

In this Letter, we demonstrate that the molecules' Ramsey dynamics are quantitatively described by an $X Y$ spin model with anisotropic $1 / r^{3}$ interactions and rule out multiple alternative models. This validation required experimental developments and extensive comparisons with theory. Experimentally, we demonstrate that we can tune the dipolar interactions (by a factor of 2) by using two different rotational state pairs, and we systematically measure the dynamics under controlled conditions to quantitatively extract dependences on molecule number. The faster dipolar dynamics was important to resolve interaction effects previously masked by experimental
PACS numbers: 67.85.-d, 03.65.Yz, 37.10.Ty, 75.10.Jm

imperfections, which play a role at long times. Furthermore, observing dynamics that is identical (up to an overall rescaling of time) for the two rotational state choices allows us to demonstrate that the effects of processes other than interactions are negligible. Theoretically, we develop and employ a cluster expansion technique, which we term the "moving-average cluster expansion" (MACE), illustrated in Fig. 1(d), that is capable of describing the relevant nonequilibrium dynamics of spin models with long range and anisotropic interactions [18].

Polar molecules and long-ranged spin models.-Polar molecules pinned in an optical lattice can realize the longranged spin- $1 / 2$ model

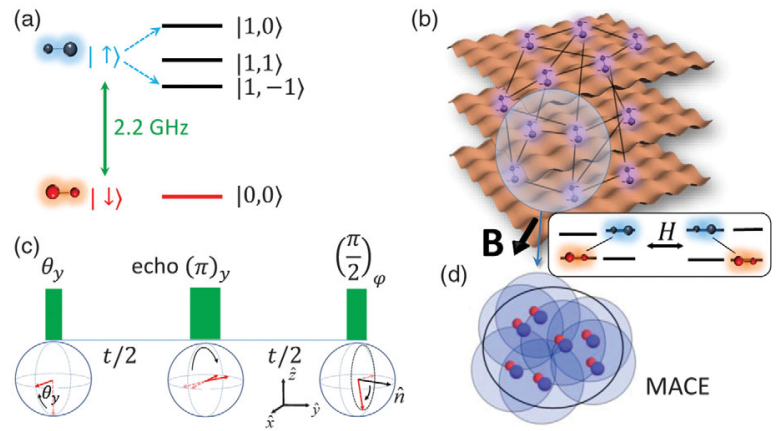

FIG. 1 (color). Probing dipolar spin-exchange interactions of molecules in a lattice with Ramsey spectroscopy. (a) Two pairs of rotational states in $\mathrm{KRb}$ molecules used to realize spin models. The states are labeled $\left|N, m_{N}\right\rangle$, where $N$ is the total rotational angular momentum and $m_{N}$ its projection along the quantization axis set by the magnetic field $\mathbf{B}$. (b) A dilute gas of $\mathrm{KRb}$ molecules pinned in a deep optical lattice and experiencing long-range, anisotropic dipolar exchange interactions. (c) Ramsey protocol applied to initiate and probe the spin dynamics. (d) Schematic of the theoretical method employed in this Letter (MACE). 


$$
H=\sum_{i \neq j} \frac{V_{i j}}{2}\left[\frac{J_{\perp}}{2}\left(S_{i}^{+} S_{j}^{-}+\text {H.c. }\right)+J_{z} S_{i}^{z} S_{j}^{z}\right],
$$

by encoding the spin in two rotational states $[16,17,19]$. (Alternative proposals to implement spin models in polar molecules are discussed in Refs. [20-28].) Here, $S_{i}^{ \pm}$and $S_{i}^{z}$ are spin- $1 / 2$ operators satisfying $\left[S_{i}^{z}, S_{j}^{ \pm}\right]= \pm \delta_{i j} S_{i}^{ \pm}$, and the sums run over all occupied lattice sites with positions $\mathbf{r}_{i}$ in units of the lattice spacing $a=532 \mathrm{~nm}$. The dipolar interaction couples spins $i$ and $j$ with strength $V_{i j}=\left(1-3 \cos ^{2} \Theta_{i j}\right) /\left|\mathbf{r}_{i}-\mathbf{r}_{j}\right|^{3}$, where $\Theta_{i j}$ is the angle between $\mathbf{r}_{i}-\mathbf{r}_{j}$ and the quantization axis, which in our system makes an angle of $45^{\circ}$ with the $\hat{X}$ and $\hat{Y}$ lattice directions [see Fig. 1(b)]. The "exchange" or " $X Y$ " terms, $S_{i}^{+} S_{j}^{-}+$H.c., swap the spin states of molecules $i$ and $j$. These arise from the transition dipole between the $|\uparrow\rangle$ and $|\downarrow\rangle$ rotational states, and they allow one molecule to flip from up to down while the other flips from down to up, together conserving their combined rotational energy. The "direct" or "Ising" terms, $S_{i}^{z} S_{j}^{z}$, arise because generally (at finite field) the dipole moments for $|\uparrow\rangle$ and $|\downarrow\rangle$ differ, and thus the parallel and antiparallel configurations of two molecules have different energies. Note that Eq. (1) does not assume unit filling or even homogeneity; we will consider the details of the experimental distributions of molecules later.

Figure 1 illustrates our experimental system, similar to that in Ref. [15], and described in detail in the Supplemental Material [18]. We create $N=6000$ to 23000 ground-state fermionic molecules of ${ }^{40} \mathrm{~K}^{87} \mathrm{Rb}$ in the lattice. These molecules have lifetimes exceeding $25 \mathrm{~s}$ in the deep 3D optical lattice [7], with the lifetime limited by off-resonant light scattering. In our experiment we work at zero electric field, where the dipole moments and thus $J_{z}$ vanish, while the resonant exchange coupling $J_{\perp}$ remains finite. However, in order to benchmark the cluster expansions we will also theoretically study the case $J_{\perp}=0, J_{z} \neq 0$.

To probe the spin system described by Eq. (1), we use a Ramsey protocol identical to the one used in Ref. [15] and illustrated in Fig. 1(c). The spins are uniformly rotated to an equal superposition state by a resonant $\pi / 2$ microwave pulse about the $\hat{y}$ spin axis, and at a later time $t$ we read out the evolution of the spins by application of a final $\pi / 2$ pulse that is phase shifted by $\varphi$. This final pulse is equivalent to rotation about a vector $\hat{\mathbf{n}}=(\sin \varphi, \cos \varphi, 0)$ and measures the quantity $\cos \varphi \sum_{i}\left\langle S_{i}^{x}\right\rangle-\sin \varphi \sum_{i}\left\langle S_{i}^{y}\right\rangle$. By varying $\varphi$, we determine the global Ramsey fringe contrast $\mathcal{C}$ defined here as

$$
C=2\left[\left\langle S_{i}^{x}\right\rangle^{2}+\left\langle S_{i}^{y}\right\rangle^{2}\right]^{1 / 2} .
$$

We include a $\pi$ spin-echo pulse around $\hat{y}$ at time $t / 2$ to remove the dephasing associated exclusively with inhomogeneous light shifts and isolate the effects of spin-spin interactions.

We vary the strength of the dipolar interactions by choosing different pairs of rotational states to realize the spin- $1 / 2$ system. The exchange coupling $J_{\perp}$ is determined by the transition matrix dipole element $d_{\uparrow \downarrow}=\langle\downarrow|d| \uparrow\rangle$, with $d$ the appropriate spherical component of the dipole operator, via $J_{\perp}=-d_{\downarrow \uparrow}^{2} / 4 \pi \epsilon_{0} a^{3}$, where $\epsilon_{0}$ is the free space permittivity [17]. For the states used in Ref. [15], $|\downarrow\rangle=$ $\left|N=0, m_{N}=0\right\rangle$ and $|\uparrow\rangle=|1,-1\rangle$, and for our lattice geometry, the transition dipole moment is expected to give an exchange frequency of $\left|J_{\perp} /(2 h)\right| \approx 52 \mathrm{~Hz}$, where $h$ is Planck's constant. For the alternative choice of rotational spin states, $|\downarrow\rangle=|0,0\rangle$ and $|\uparrow\rangle=|1,0\rangle,\left|J_{\perp} /(2 h)\right|$ is predicted to be twice as strong $(\sim 100 \mathrm{~Hz}$; we neglect the small differences in hyperfine admixing effects between the rotational states [15]). This enhancement occurs because the molecules are aligned and oscillate along the quantization axis, as opposed to rotating about it, so that the dipole coupling is not reduced through time averaging [17,29].

Figure 2 compares the observed contrast dynamics as a function of evolution time $t$ for three different molecule numbers, $N \sim 5 \times 10^{3}, 1 \times 10^{4}$, and $2 \times 10^{4}$, and both pairs of rotational states. A larger coupling is observed for the $\{|0,0\rangle,|1,0\rangle\}$ pair, and the faster coherent spin dynamics reduces the effects of technical limitations on the coherence times. As will be detailed below, the theory-experiment agreement for different exchange couplings and filling fractions further validates the experimental realization of the spin model in Eq. (1).

Theory.-To simulate the dynamics governed by Eq. (1), we develop a novel cluster expansion technique. We compute $\left\langle S_{i}^{\alpha}(t)\right\rangle(\alpha=\{x, y, z\})$ by building an optimal cluster for spin $i$ containing the spins connected to it by the largest coupling constants $V_{i j}$; see Fig. 1(d). The dynamics of $\left\langle S_{i}^{\alpha}(t)\right\rangle$ is then computed by exactly solving the dynamics of the entire associated cluster numerically, and the contrast is obtained by summing these spin expectation values over all $i$. The MACE method is rather robust to artifacts arising from finite cluster sizes; by constructing an optimal cluster for each spin, the MACE reduces surface contributions where the dynamics of boundary spins would not be accurately captured. We show results for size $g=10$ clusters, where the method is converged for the system shown here. The convergence of the method is analyzed in the Supplemental Material [18], where we vary the cluster size for the present problem as well as by applying the MACE to the Ising limit $\left(J^{z} \neq 0\right.$, $J^{\perp}=0$ ), where an analytic solution exists [12,30-32].

We choose a molecule distribution according to our rough expectations of the experimental distribution based on the molecule formation process. In particular, the molecules are produced only at sites of the lattice initially populated by exactly one $\mathrm{Rb}$ and one $\mathrm{K}$ atom. Guided by the initial atomic numbers, temperatures, and trapping parameters [18], we expect a doubly occupied Mott insulator domain of Rb atoms in the center of the trap, surrounded by a unit-filled ellipsoidal Mott shell where molecules may be formed. Therefore, we assume a shell of molecules with a filling probability on site $i$ given by $f_{i} \propto e^{-\left[\left(\sqrt{x_{i}^{2}+y_{i}^{2}+\alpha^{2} z_{i}^{2}}-R_{c}\right)^{2}\right] /\left(2 w^{2}\right)}$, with $\alpha=7$ reflecting the ratio of axial and radial trapping 


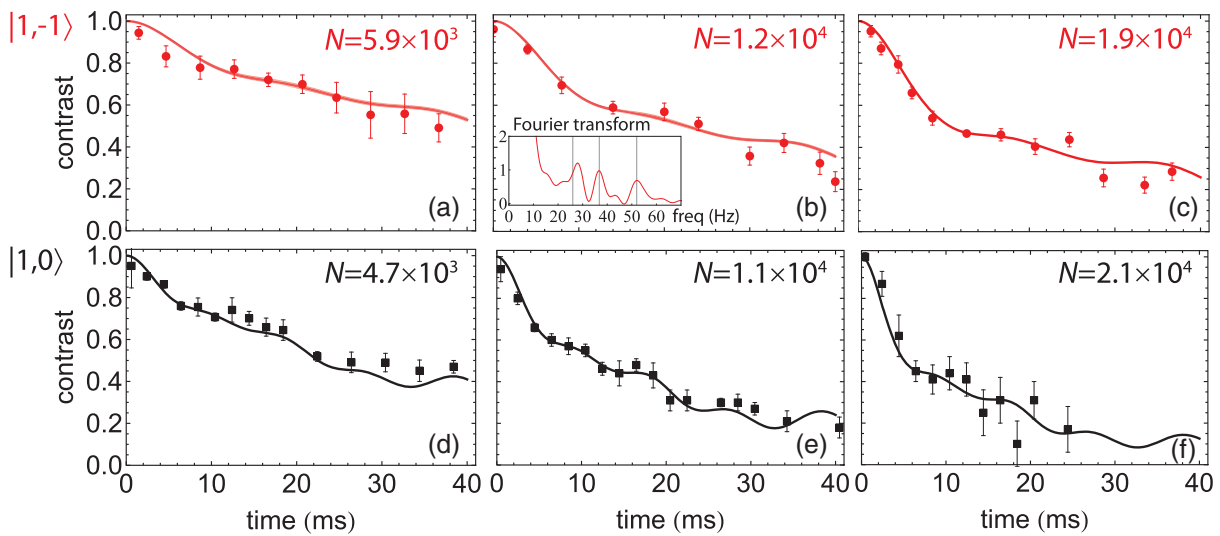

FIG. 2 (color). Measured contrast dynamics compared with theory. (a)-(c) Contrast versus time for the $\{|\uparrow\rangle=|1,-1\rangle,|\downarrow\rangle=|0,0\rangle\}$ rotational state choice, experimentally measured (red symbol) and theoretically calculated (red line) for all spins initially prepared along $\hat{x}$, for increasing molecule number $N$ (left to right). Inset: Fourier transform of the simulated dynamics for $N=1.2 \times 10^{4}$ and $|\uparrow\rangle=|1,-1\rangle$. (d)-(f) Black symbols and black line, same as in (a)-(c), but choosing instead $|\uparrow\rangle=|1,0\rangle$.

frequencies, and the central shell radius of $R_{c}=35$. We choose whether site $i$ is occupied randomly with probability $f_{i}$. Although this generates a disordered configuration, the dynamics is self-averaging and varies little from configuration to configuration (shown below). We determine the filling fraction by varying the shell width $w$, and set it to match the experimentally observed spin-echo contrast decay time for $N=1.2 \times 10^{4}$ molecules. Using this procedure, we find $w=30$. Although our calculations use this specific distribution, we find that for a fixed local peak filling of molecules our theoretical results are largely independent of the chosen geometry.

Comparing theory and experiment.-Figure 2 demonstrates that our calculations (solid lines) quantitatively agree with the measurements for both rotational state choices and for a broad range of densities and evolution times. A small shaded band around the lines indicates the standard deviation due to shot-to-shot fluctuations of the molecule configuration. We emphasize that our results use a single global parameter to reproduce all the experimental data. This parameter is the ratio of the molecule filling factor $f$ to the molecule number $N$.

We note a few interesting trends that emerge in both the theory and experimental data, then describe in the following paragraphs each of these trends in more detail. First, as in Ref. [15], we observe clear oscillations of the contrast. These oscillations are roughly independent of the molecule number $N$, but the frequency is found to be larger for the $|1,0\rangle$ data, consistent with the enhanced spin-exchange coupling. Second, we observe that the spin coherence time decreases with an increase in lattice filling. This is a clear signature of spin-spin interactions. In comparing panels (a)-(c) with (d)-(f) of Fig. 2, this coherence time is also seen to be shorter for the spin states with larger spin-exchange coupling $(|\uparrow\rangle \equiv|1,0\rangle)$. Lastly, we find a trend of increasing oscillation amplitude for increasing molecule number.

The contrast oscillations arise from dipole-dipole interactions between the molecules. Clear oscillations at frequency $\left|J_{\perp} /(2 h)\right|$ are visible for all measurements and calculations. Additionally, the Fourier transform of the theoretical contrast (Fig. 2, inset) clearly shows multiple oscillation frequencies on a broad, structured background. These frequencies are roughly determined by the size of the strongest couplings. The most prominent contributions apparent in the time evolution data appear at the frequencies $\nu, \nu / \sqrt{2}$, and $\nu / 2$ (next nearest neighbor coupling strengths), where $\nu=104 \mathrm{~Hz}$ for $|\uparrow\rangle=|1,0\rangle$ data and $52 \mathrm{~Hz}$ for $|\uparrow\rangle=|1,-1\rangle$. We have analyzed the experimental data by fitting to functional forms that oscillate at either a single frequency $\nu$ or at three frequencies [18]. Based on a global analysis of fifteen data sets, we find clear evidence that a multifrequency fit better captures the observed dynamics. Moreover, the analysis suggests a fundamental frequency of $\nu \sim 108 \mathrm{~Hz}$ for the $|\uparrow\rangle=$ $|1,0\rangle$ data (reduced by half for the $|1,-1\rangle$ ), in excellent agreement with the expected value. The influence of the choice of rotational states is further illustrated in Fig. 3(a). This plot overlays contrast dynamics for the two pairs of rotational states, with the times of the $|\uparrow\rangle=|1,-1\rangle$ data rescaled by a factor of $1 / 2$ to account for the different dipolar interaction strength. The collapse of the two data sets highlights that all of the observed dynamics arise from the dipolar interactions.

To investigate the coherence time $\tau$ 's dependence on the particle number $N$, we experimentally reduce the number of molecules using single-particle loss due to off-resonant light scattering. This leaves the distribution of molecules invariant by reducing the density of particles uniformly [18]. We extract coherence times by fitting the contrast dynamics to $\exp (-t / \tau)$ for both the theory and experiment; the results are shown in Fig. 3(b). Whereas the oscillations come mainly from the largest frequencies (smallest spacings) between molecules, the decoherence arises from interactions of molecules at a variety of spacings. We expect $\tau \propto 1 / N$, which is a characteristic signature of interactions. The scaling arises because, as the lattice filling $f \propto N$ increases, the mean distance between molecules decreases as $\bar{R} \sim f^{-1 / 3}$, leading to an average dipolar interaction that 

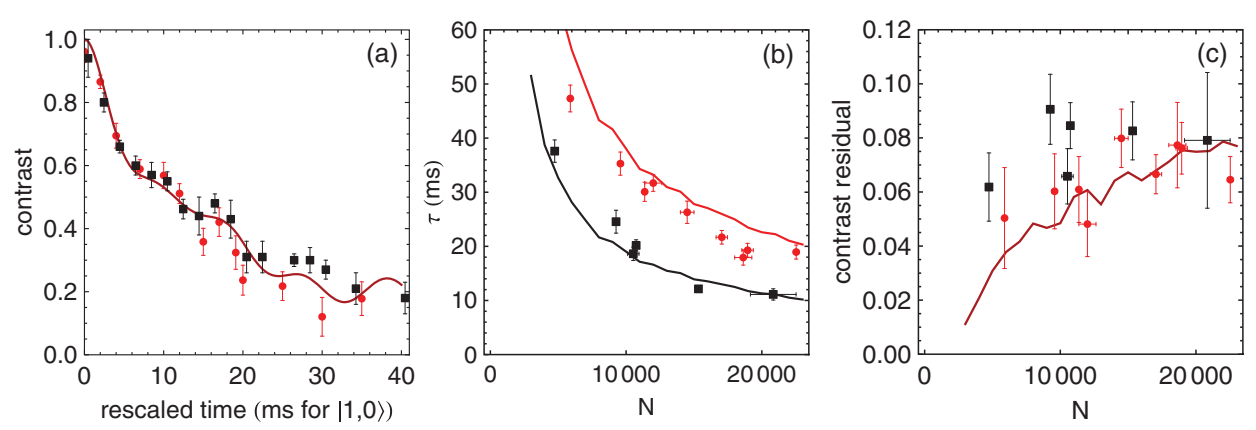

FIG. 3 (color). Contrast dynamics' dependence on particle number (lattice filling) and rotational state pair choice. (a) Rescaled contrast dynamics for the two choices of spin states: the experimental times for the $|\uparrow\rangle=|1,-1\rangle$ data (red circles) are rescaled by a factor of $J_{|1,-1\rangle} / J_{|1,0\rangle} \approx 1 / 2$ with respect to the $|\uparrow\rangle=|1,0\rangle$ data (black squares), to show that the choice of rotational states only rescales the interaction timescale. Theoretically calculated dynamics (solid line) is shown for $N=12,000$. The two measurements have slightly different particle numbers: $N=1.1 \times 10^{4}$ and $N=1.2 \times 10^{4}$ for $|\uparrow\rangle=|1,0\rangle$ and $|\uparrow\rangle=|1,-1\rangle$, respectively. (b) Spin coherence time $\tau$ versus molecule number $N$, as determined by fitting the contrast data to a simple exponential decay, of the form $C(t)=\exp (-t / \tau)$. (c) Root-mean-square residuals of the data from the exponential fit as a function of the molecule number $N$, quantifying the magnitude of all oscillations. Coherence times and residuals are shown for both sets of spin states, with theory predictions as well, with the same colors and symbols as in panel a.

scales as $1 / \bar{R}^{3} \sim f$. We indeed see an approximate scaling $\tau \propto 1 / N$, as well as a factor of 2 reduction in coherence time for the $|1,0\rangle$ data compared to the $|1,-1\rangle$ data. We note that the theory here uses a peak filling of $f=8 \%$ for a molecule number of $N=2 \times 10^{4}$, which is within a factor of 2 of estimates based on loss measurements $(\sim 9 \%$ for $N \sim 1 \times 10^{4}$ ) and direct imaging [15,33].

Finally, the oscillation amplitude increases with $N$ for the experimental fillings studied since the probability of a molecule having an occupied nearest neighbor site increases with $N$. To characterize the amplitude of the oscillations, we plot in Fig. 3(c) the root-mean-square (rms) residuals of the data from the exponential fit. We find that for increasing molecule number $N$ there is a systematic increase of the residuals, due to oscillatory dynamics absent in the simple exponential fit.

We find that the dynamics is sensitive to the microscopic form of the Hamiltonian and consequently our measurements can rule out multiple alternatives to the spin-exchange model Eq. (1) for describing the experimental observations. For example, the experimentally measured dynamics is inconsistent with the Ising model, where the contrast
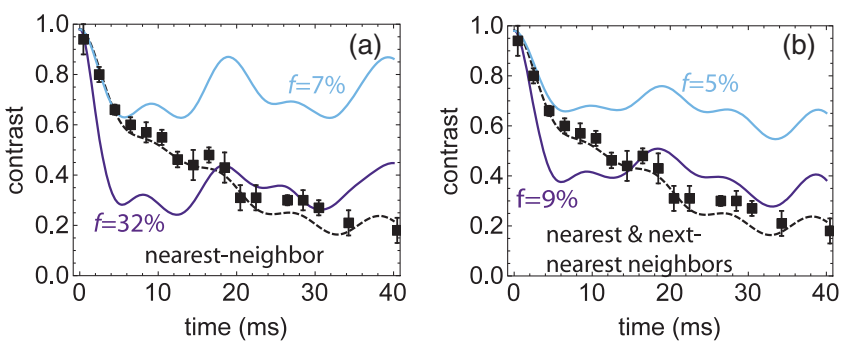

FIG. 4 (color). Theoretically calculated contrast dynamics for (a) nearest-neighbor interactions and (b) interactions to nextnearest-neighbors for two fillings each (indicated) compared with the $N=1.2 \times 10^{4},|\uparrow\rangle=|1,0\rangle$ measurement and dipolar theory (dashed line). The disagreement of the short-ranged calculations with experiment supports the necessity of including long-range interactions to describe the observed dynamics. oscillation amplitudes are significantly smaller than those observed experimentally (see Fig. S1 in the Supplemental Material [18].) Also, as shown in Fig. 4, the MACE calculations of the spin-exchange model dynamics with interactions truncated to nearest or next nearest neighbors in the lattice are inconsistent with our measurements, pointing to the long-ranged nature of the interactions.

Outlook. - The experimental developments presented here further the ability of ultracold-molecule experiments to harness effective spin interactions using rotational states by enabling larger, more tunable interaction strengths. The comparison with theory demonstrates that the observable physics is quantitatively governed by the spin model Eq. (1). Our experimental and theoretical results may provide insight into other systems described by long-range spin models [34-47]—for example, magnetic atoms, Rydberg atoms, trapped ions, and excitons in solid state materials and molecules. In the future it will be fascinating to examine the development of correlations more directly and to explore transport and thermalization, or lack thereof, e.g., glassiness and many-body localization [48-51].

We thank Bihui Zhu, Salvatore Manmana, Tilman Pfau, John Bollinger, Joe Britton, Brian Sawyer, Johannes Schachenmayer, Jake Taylor, and Alexey Gorshkov for discussions. The authors acknowledge funding from JILANSF-PFC 1125844, NSF-PIF, ARO, ARO-DARPA-OLE, and AFOSR. S. A. M. and J.P.C. acknowledge funding from NDSEG. K. R. A. H., B. G., and M. F.-F. thank the National Research Council for support. K. R. A. H. and A. M. R. acknowledge the KITP.

*kaden.hazzard@colorado.edu

[1] K.-K. Ni, S. Ospelkaus, M. H. G. de Miranda, A. Pe'er, B. Neyenhuis, J. J. Zirbel, S. Kotochigova, P. S. Julienne, D. S. Jin, and J. Ye, Science 322, 231 (2008). 
[2] K.-K. Ni, S. Ospelkaus, D. Wang, G. Quéméner, B. Neyenhuis, M. H. G. de Miranda, J. L. Bohn, J. Ye, and D. S. Jin, Nature (London) 464, 1324 (2010).

[3] S. Ospelkaus, K.-K. Ni, D. Wang, M. H. G. de Miranda, B. Neyenhuis, G. Quéméner, P. S. Julienne, J. L. Bohn, D. S. Jin, and J. Ye, Science 327, 853 (2010).

[4] M. H. G. de Miranda, A. Chotia, B. Neyenhuis, D. Wang, G. Quéméner, S. Ospelkaus, J. L. Bohn, J. Ye, and D. S. Jin, Nat. Phys. 7, 502 (2011).

[5] S. Ospelkaus, K.-K. Ni, G. Quéméner, B. Neyenhuis, D. Wang, M. H. G. de Miranda, J.L. Bohn, J. Ye, and D. S. Jin, Phys. Rev. Lett. 104, 030402 (2010).

[6] B. Neyenhuis, B. Yan, S. A. Moses, J. P. Covey, A. Chotia, A. Petrov, S. Kotochigova, J. Ye, and D. S. Jin, Phys. Rev. Lett. 109, 230403 (2012).

[7] A. Chotia, B. Neyenhuis, S. A. Moses, B. Yan, J. P. Covey, M. Foss-Feig, A. M. Rey, D. S. Jin, and J. Ye, Phys. Rev. Lett. 108, 080405 (2012).

[8] M. Baranov, Phys. Rep. 464, 71 (2008).

[9] G. Pupillo, A. Micheli, H. Büchler, and P. Zoller, in Cold Molecules: Creation and Applications, edited by R. V. Krems, B. Friedrich, and W. C. Stwalley (Taylor \& Francis, London, 2008).

[10] L. D. Carr, D. DeMille, R. V. Krems, and J. Ye, New J. Phys. 11, 055049 (2009).

[11] M. Lemeshko, R. V. Krems, J. M. Doyle, and S. Kais, Mol. Phys. 111, 1648 (2013).

[12] K. R. A. Hazzard, S. R. Manmana, M. Foss-Feig, and A. M. Rey, Phys. Rev. Lett. 110, 075301 (2013).

[13] A. Pikovski, M. Klawunn, A. Recati, and L. Santos, Phys. Rev. A 84, 061605(R) (2011).

[14] D. DeMille, Phys. Rev. Lett. 88, 067901 (2002).

[15] B. Yan, S. A. Moses, B. Gadway, J. P. Covey, K. R. A. Hazzard, A. M. Rey, D. S. Jin, and J. Ye, Nature (London) 501, 521 (2013).

[16] R. Barnett, D. Petrov, M. Lukin, and E. Demler, Phys. Rev. Lett. 96, 190401 (2006).

[17] A. V. Gorshkov, S. R. Manmana, G. Chen, J. Ye, E. Demler, M. D. Lukin, and A. M. Rey, Phys. Rev. Lett. 107, 115301 (2011).

[18] See Supplemental Material at http://link.aps.org/ supplemental/10.1103/PhysRevLett.113.195302 for a description of the experimental production of the ultracold molecules and their trapping in a lattice, analysis of the experimental data to test for multiple oscillation frequencies, and convergence studies of the theoretical methods.

[19] S. R. Manmana, E. M. Stoudenmire, K. R. A. Hazzard, A. M. Rey, and A. V. Gorshkov, Phys. Rev. B 87, 081106 (2013).

[20] A. Micheli, G. Brennen, and P. Zoller, Nat. Phys. 2, 341 (2006).

[21] H. P. Büchler, A. Micheli, and P. Zoller, Nat. Phys. 3, 726 (2007).

[22] T. Watanabe, Phys. Rev. A 80, 053621 (2009).

[23] M. L. Wall and L. D. Carr, New J. Phys. 11, 055027 (2009).

[24] Cold Molecules: Creation and Applications, edited by R. V. Krems, B. Friedrich, and W. C. Stwalley (Taylor \& Francis, London, 2008).

[25] J. Schachenmayer, I. Lesanovsky, A. Micheli, and A. J. Daley, New J. Phys. 12, 103044 (2010).
[26] J. Pérez-Ríos, F. Herrera, and R. V. Krems, New J. Phys. 12, 103007 (2010).

[27] C. Trefzger, M. Alloing, C. Menotti, F. Dubin, and M. Lewenstein, New J. Phys. 12, 093008 (2010).

[28] J. P. Kestner, B. Wang, J. D. Sau, and S. Das Sarma, Phys. Rev. B 83, 174409 (2011).

[29] A. V. Gorshkov, P. Rabl, G. Pupillo, A. Micheli, P. Zoller, M. D. Lukin, and H. P. Büchler, Phys. Rev. Lett. 101, 073201 (2008).

[30] G. G. Emch, J. Math. Phys. (N.Y.) 7, 1198 (1966).

[31] M. Kastner, Phys. Rev. Lett. 106, 130601 (2011).

[32] M. Foss-Feig, K. R. A. Hazzard, J. J. Bollinger, and A. M. Rey, Phys. Rev. A 87, 042101 (2013).

[33] B. Zhu, B. Gadway, M. Foss-Feig, J. Schachenmayer, M. Wall, K. R. A. Hazzard, B. Yan, S. A. Moses, J. P. Covey, D. S. Jin, J. Ye, M. Holland, and A. M. Rey, Phys. Rev. Lett. 112, 070404 (2014).

[34] G. A. Álvarez and D. Suter, Phys. Rev. Lett. 104, 230403 (2010).

[35] D. E. Chang, J. Ye, and M. D. Lukin, Phys. Rev. A 69, 023810 (2004).

[36] M. J. Martin, M. Bishof, M. D. Swallows, X. Zhang, C. Benko, J. von Stecher, A. V. Gorshkov, A. M. Rey, and J. Ye, Science 341, 632 (2013).

[37] T. Lahaye, T. Koch, B. Fröhlich, M. Fattori, J. Metz, A. Griesmaier, S. Giovanazzi, and T. Pfau, Nature (London) 448, 672 (2007).

[38] T. Lahaye, C. Menotti, L. Santos, M. Lewenstein, and T. Pfau, Rep. Prog. Phys. 72, 126401 (2009).

[39] M. Saffman, T. G. Walker, and K. Mølmer, Rev. Mod. Phys. 82, 2313 (2010).

[40] B. P. Lanyon, C. Hempel, D. Nigg, M. Mller, R. Gerritsma, F. Zhringer, P. Schindler, J. T. Barreiro, M. Rambach, G. Kirchmair, M. Hennrich, P. Zoller, R. Blatt, and C. F. Roos, Science 334, 57 (2011).

[41] J. Nipper, J. B. Balewski, A. T. Krupp, S. Hofferberth, R. Löw, and T. Pfau, Phys. Rev. X 2, 031011 (2012).

[42] M. Lu, N. Q. Burdick, and B. L. Lev, Phys. Rev. Lett. 108, 215301 (2012).

[43] A. de Paz, A. Sharma, A. Chotia, E. Maréchal, J. H. Huckans, P. Pedri, L. Santos, O. Gorceix, L. Vernac, and B. Laburthe-Tolra, Phys. Rev. Lett. 111, 185305 (2013).

[44] J. W. Britton, B. C. Sawyer, A. C. Keith, C.-C. J. Wang, J. K. Freericks, H. Uys, M. J. Biercuk, and J. J. Bollinger, Nature (London) 484, 489 (2012).

[45] R. Islam, C. Senko, W. C. Campbell, S. Korenblit, J. Smith, A. Lee, E. E. Edwards, C.-C. J. Wang, J. K. Freericks, and C. Monroe, Science 340, 583 (2013).

[46] P. Xiang, M. Litinskaya, and R. V. Krems, Phys. Rev. A 85, 061401 (2012).

[47] S. K. Saikin, A. Eisfeld, S. Valleau, and A. Aspuru-Guzik, Nanophotonics 2, 21 (2013).

[48] D. M. Basko, I. L. Aleiner, and B. L. Altshuler, Ann. Phys. (Amsterdam) 321, 1126 (2006).

[49] A. Pal and D. A. Huse, Phys. Rev. B 82, 174411 (2010).

[50] M. P. Kwasigroch and N. R. Cooper, Phys. Rev. A 90, 021605 (2014).

[51] N. Y. Yao, C. R. Laumann, S. Gopalakrishnan, M. Knap, M. Mueller, E. A. Demler, and M. D. Lukin, arXiv: 1311.7151. 